Positions moyennes des étoiles de comparaison.

\begin{tabular}{|c|c|c|c|}
\hline *: & re $1909.0,10.0$ & $\delta \mathrm{r} 909.0,10.0$ & Autorité \\
\hline $\mathbf{I}$ & $2^{\mathrm{h}} 24^{\mathrm{m}} 25^{\mathrm{s}} \cdot 34$ & $+x x^{\circ} 5^{1^{\prime}} 20^{\prime \prime} 4$ & AG Lpz I 724 \\
\hline 2 & 2 I0 6.04 & $\begin{array}{lll}\operatorname{II} & 18 & 46.4\end{array}$ & 660 \\
\hline 3 & I 394.59 & +94335.5 & AG Lpz II 649 \\
\hline 4 & I $3348.1 \mathrm{I}$ & +92626.0 & 6 I 4 \\
\hline 5 & $\circ 5748.53$ & $+820 \mathrm{r} 7.1$ & 367 \\
\hline 6 & $\begin{array}{lll}50 & 0.20\end{array}$ & +853.0 & 308 \\
\hline 7 & - $46 \quad 23.55$ & +75048.4 & 286 \\
\hline 8 & - $44 \quad 56.42$ & +7527.5 & 272 \\
\hline
\end{tabular}

Remarques. La plupart des observations de la comète de Halley ont été faites dans des conditions atmosphériques particulièrement défavorables et souvent pendant une eclaircie ou à travers des bandes de nuages. Grossissement 90 fois. La grandeur a été estimée d'après celle de l'étoile de comparaison et des étoiles du voisinage. Elle se rapporte au noyau car la queue n'est devenue visible que

Geneve, 25 avril 19 Io.

\begin{tabular}{|c|c|c|c|}
\hline$*$ & $\alpha \quad 1910.0$ & $\delta \times 910.0$ & Autorité \\
\hline 9 & $0^{h} 42^{m} 13^{s} \cdot 7$ I & $+7^{\circ} 48^{\prime} 57^{\prime \prime} .5$ & AG Lpz II 257 \\
\hline 10 & $\circ \quad 34 \quad 19.27$ & +7594.3 & Toul ph Cliché 5 No. 9 \\
\hline I I & $\circ 3348.90$ & +74651.4 & AG Lpz II I 99 \\
\hline $\mathrm{I} 2$ & 03342.89 & $+75^{8} 32.1$ & I 97 \\
\hline$x_{3}$ & 03051.03 & +7396.0 & $18 \mathrm{I}$ \\
\hline I 4 & $0 \quad 2940.28$ & +8 I I 21.9 & 176 \\
\hline$\times 5$ & $0255^{2.42}$ & $+8 \circ 54.8$ & r 50 \\
\hline I 6 & $235 \mathrm{I}$ & $+7432 \mathrm{I} . \mathrm{I}$ & I I 8 I 8 \\
\hline
\end{tabular}

dans les observations des 8 et 9 mars. Le 23 avril, le noyau est allongé et se prolonge en une queue d'environ ro minutes de longueur. Des clichés photographiques avec pose d'une heure entière ont été pris le 30 décembre, les I 3 et 16 janvier, les 5 et 16 février. Avec une pose plus courte les 5 et 20 février, 5 et 9 mars et le 23 avril.

F. Pidoux, astronome.

Observations of (8) Flora.

\begin{tabular}{|c|c|c|c|c|c|c|c|}
\hline $1909-10$ & Gr. M. T. & $A \alpha$ & $A \delta$ & $\alpha$ app. & $\delta$ app. & Red. ad l. app. & $*$ \\
\hline Dec. I 6 & $18^{\mathrm{h}} 30^{\mathrm{m}} \mathrm{o}^{\mathrm{s}}$ & $-\mathrm{I}^{\mathrm{m}} 24^{\mathrm{s}} .18$ & $-12^{\prime} 21^{\prime \prime} 7$ & $3^{\mathrm{h}} 15^{\mathrm{m}} \quad 0^{\mathrm{s}} \cdot 4^{6}$ & $+10^{\circ} 6^{\prime} 5^{\prime \prime} \mathrm{I}$ & $+3^{\mathrm{s}} \times 9+13^{\prime \prime} 8$ & $\mathbf{I}$ \\
\hline Jan. Io & I $5 \quad 324$ & $+2 \quad 1.96$ & -246.7 & $\begin{array}{lll}3 & 17 & 7.89\end{array}$ & $+\mathrm{I} 25 \mathrm{I}$ III. & $-0.21+0.3$ & 2 \\
\hline Febr. 5 & $1534 \quad 10$ & -134.12 & $0 \quad 0.0$ & $340 \quad 53 \cdot 3^{6}$ & +162542.5 & $-0.3^{8}+$ I.I & 3 \\
\hline I 2 & $16 \quad 4 \quad 33$ & 7.92 & $+\quad$ I 25.3 & 350 & +172337.4 & $-0.45+\mathrm{I} .5$ & 4 \\
\hline
\end{tabular}

Mean places of the comparison stars.

\begin{tabular}{|c|c|c|c|c|c|c|c|}
\hline$: ;$ & $\alpha$ I 909.0, I 0.0 & $\delta$ I 909.0, I0.0 & Authority & $\%$ & $\alpha$ I9 10.0 & $\delta$ rgro.o & Authority \\
\hline I & $3^{\mathrm{h}} \mathrm{I} 6^{\mathrm{m}} 2 \mathrm{I}^{\mathrm{s}} \cdot 45$ & $+10^{\circ} \mathrm{I} 8^{\prime} \mathrm{I} 3^{\prime \prime} \mathrm{O}$ & $\mathrm{BD}+10^{\circ} 43^{2}$ & 3 & $3^{\mathrm{h}} 42^{\mathrm{m}} 27^{\S}: 86$ & $+16^{\circ} 25^{\prime} 4 I^{\prime \prime} 4$ & AG Berl A Iого \\
\hline 2 & 3.156 .14 & + I 25357.6 & $\begin{array}{l}\mathrm{BD}+\mathrm{r}^{\circ}{ }^{\circ} 68, \text { ref. to } \mathrm{AG} \\
\mathrm{Lpz} \mathrm{I} 989\end{array}$ & 4 & $\begin{array}{lll}3 & 50 & \text { I } 7.08\end{array}$ & $+1722 \quad 10.6$ & AG Berl A 1048 \\
\hline
\end{tabular}

The position of star I was measured on my plates by Professor E. E. Barnard of Yerkes Observatory. Hè also made the reductions of his measurements.

Des Moines, Iowa, r 9 ro March 2 r.

D. W. Morehouse.

\title{
Observations of the Minor Planet (403) Cyane.
}

\section{By E. E. Barnard.}

On turning the telescope to the variable star RS Aquarii $\mid$ of the same brightness as star r. Motion soon showed it July I. 2 , I was surprised to see a strange star near the variable. It lay nearly midway between the stars 1 and 2 of the diagram given in A. N. 4 I $5^{\circ}$ p. 337 and was very nearly to be an asteroid moving to the west. The following measures were obtained of it with the 40 -inch micrometer. The times are $6^{\mathrm{h}} \mathrm{o}^{\mathrm{m}}$ slow of Gr. M. T.

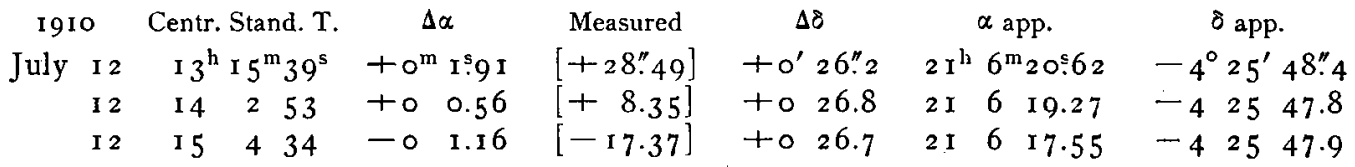

$$
\begin{aligned}
& \alpha \quad \delta \quad \text { Red.ad 1.app. Authority } \\
& 2 I^{\text {h }} 6^{\mathrm{m}} \text { I } 6.66-4^{\circ} 26^{\prime} \text { I } 8^{\prime \prime} 6 \quad+2.05+4^{\prime \prime} \circ \quad \text { E. E. Barnard, A. N. } 4 \text { I } 50 \text { p. } 343 \text { star } 2
\end{aligned}
$$


By careful comparison with star $x$ (of the 13.04 magnitude, A. N. 4 I 50) the magnitude of the asteroid.was I3.I I. The color was apparently white.

Yerkes Observatory, Williams Bay, Wisconsin, r 9 ro July I 3.

The only asteroid near this place given in B. J. is (403) Cyane, whose opposition occurs I9 ro August 3. I have assumed that the object is Cyane.

PS. July r 4. Since sending the above observations yesterday, A. N. 4423 came to day and in it is an ephemeris of Cyane which shows it to be the object observed by me.

\title{
Über die Veränderlichkeit von $60 \mathrm{~B}$. Canum venaticorum.
}

\author{
(Auszug aus Harvard Zirkular I59)
}

Bei der Überlagerung der Platten von Nr. I 6 der Harvard-Karte ergab sich, daß der von Bode als 60 Canum venaticorum bezeichnete Stern $\mathrm{BD}+{ }_{4} 6^{\circ}{ }^{1} 8$ I 7 I 905 März I 5 erheblich schwächer war als 1907 April 2. Der Stern kommt, soweit bekannt, in keinem Kataloge veränderlicher Sterne vor.*) Die Veränderlichkeit dieses Sternes wurde zuerst von Schmidt vermutet, A. N. 82.1 25, der ihn im August I872 von kaum $6^{\mathrm{m}}$ fand und dann eine Lichtzunahme bis Mitte Dezember beobachtete. I873 Juni I 7 war der Stern wieder nahe beim Minimum. A. N. 89.165 teilt Schmidt 4 Maxima und 4 Minima aus der Zeit $1873_{-1876} \mathrm{mit}$, die eine sehr unregelmäßige, genähert 386 Tage betragende Periode ergeben. Durch Luizet wurde die Veränderlichkeit bestätigt und als Grenzen derselben $5^{m_{2}}$ und $6^{m} \cdot 0$ zwischen 1898 und 1903 angegeben (A. N. I65.343).

Um eine weitere Bestätigung der Veränderlichkeit und eine Vorstellung von der Natur der Lichtkurve zu erhalten, wurde von Prof. E.C. Pickering die Helligkeit dieses Sternes

I 9 I $\circ$ März 3 I.

auf I 54 Aufnahmen zwischen r899 März I 2 und r9 10 Jan. I 5 festgestellt. Die Helligkeit der Vergleichsterne wurde durch direkte Vergleichung mit dem in der Nähe des Poles ausgewählten Normal-Größen-Satze bestimmt, indem eine auf den Pol exponierte Platte auch für die gleiche Zeit auf die Umgegend von 60 B. Can. ven. exponiert wurde.

Die Beobachtungen ergeben eine sehr unregelmäßige Veränderlichkeit.

\begin{tabular}{|c|c|c|c|c|c|}
\hline \multicolumn{6}{|c|}{ Genäherte Zeiten } \\
\hline \multicolumn{3}{|c|}{ des Maximums } & \multicolumn{3}{|c|}{ des Minimums } \\
\hline 900 & Febr. & Gr. $7^{\mathrm{m}} \cdot 7$ & I $900 \mathrm{Mai}$ & 2 & Gr. $8^{r}$ \\
\hline 01 & Juni I 2 & $7 \cdot 5$ & I90I März & I I & 0 \\
\hline & April I 5 & $7 \cdot 7$ & I $902 \mathrm{Jan}$. & I 5 & \\
\hline 10 & Febr. I 4 & 7.9 & I 906 März & 25 & \\
\hline be & Juni & $7 \cdot 5$ & & & \\
\hline
\end{tabular}

Das Spektrum des Sternes gehört zum Typus IV, Klasse $\mathrm{N}$; wegen der Färbung ist der Stern photographisch wenigstens 2 Größenklassen schwächer als visuell.

$K b$.

*) Erwähnt ist der Stern im Vorwort zu Schönfeld, Zweiter Katalog von veränderlichen Sternen Seite 4 Nr. 9. Er ist aufgeführt in Gore, Catalogue of suspected Variables als Nr. 383 und in Fr. Kriiger, Katalog der farbigen Sterne als Nr. roo3. Am letzteren Orte finden sich auch Angaben über Helligkeit, Farbe und Spektrum.

\section{Beobachtung des Sternes Grb 1830.}

Wie voriges Jahr (A. N. 4342) wurde der Stern auch heuer beobachtet und zwar:

\begin{tabular}{|c|c|c|c|c|c|}
\hline 1910 & $\alpha_{1910.0}$ & $\delta 1910.0$ & 1920 & $\alpha 1910.0$ & 81910.0 \\
\hline April & II $I^{\mathrm{h}} 47^{\mathrm{m}} 47^{\mathrm{s}} \cdot 65$ & $+38^{\circ}=1^{\prime} 49^{\prime \prime} 8$ & April 2 I & I I ${ }^{\mathrm{h}} 47^{\mathrm{m}} 47^{\mathrm{s}}: 84$ & $+38^{\circ} 21^{\prime} 52^{\prime \prime} .0$ \\
\hline $\mathbf{I}$ & 47.87 & $5 I . I$ & 22 & 47.68 & $49 \cdot 4$ \\
\hline 20 & 47.75 & $5^{0.4}$ & & & \\
\hline
\end{tabular}

Beobachter: K. Koss. Bei der Reduktion auf I9 10.0 wurde die Eigenbewegung aus Newcombs Catalogue of Fundamental Stars angewendet.

Pola, am 30. April 1910.

Der Vorstand: Koss, k. u. k. Fregattenkapitän.

Erratazcorrige. In "Bonner Sternverzeichnis IV. Section (Schönfeld)" per la stella - r $9^{\circ} 5057$ invece di $\delta=-19^{\circ} 33^{\prime} \cdot 5$ deve leggersi $\delta=-19^{\circ} 33^{\prime} \cdot \circ$, o, tutt'al più, $\delta=-19^{\circ} 33^{\prime} \cdot 1 . \quad L$. Volta.

Komet 1892 VI. Die Bearbeitung dieses Kometen ist von Herrn A. Patterer, Graz, übernommen.

Komet 1910 b (Metcalf). Die in Nr. 4434 mitgeteilten Beobachtungen von Aug. Io und Aug. I 2 (2. Beob.) sind am U. St. Nav. Obs. Washington, bez. am Stụd. Astr. Laboratory Cambridge Mass. erhalten.

Inhalt zu Nr. 4436. H. E. Lau. Mikrometermessungen von Doppelsternen. 313. - M. Ebell. Ephemeride des Halleyschen Kometen Igog c. 323. - F. Pidoux. Observations de la Comète de Halley (Igog c). 323. - D. W. Morehouse. Observations of (8) Flora. 325. - E. E. Barnarl. Observations of the. Minor Planet (403) Cyane. 325. - Über die Veränderlichkeit von 6o B. Canum venaticorum. 327. - K. Koss. Beobachtung des Sternes Grb 1830. 327. - Kleine Mitteilungen. 327.

Geschlossen rgro Aug. 24. Herausgeber H. Kobold. Druck von C. Schaidt (Georg Oheim, Alfred Oheim). Expedition: Kiel, MIoltkestr. Bo. 